\title{
Primary Bladder Adenocarcinoma: A Five-Year Retrospective Clinicopathologic Study of 42 Cases in Egyptian NCI (2010-14)
}

\author{
Yahia Ismail $^{1 *}$, Amr Kamal' ${ }^{2}$, Osama Yousof ${ }^{3}$, Neveen Tahoun ${ }^{4}$, Rasha Allam ${ }^{5}$ \\ ${ }^{1}$ Medical Oncology Department, National Cancer Institute, Cairo University, Cairo, Egypt \\ ${ }^{2}$ Surgical Oncology Department, National Cancer Institute, Cairo University, Cairo, Egypt \\ ${ }^{3}$ Radiation Oncology and Nuclear Medicine Department, National Cancer Institute, Cairo University, Cairo, Egypt \\ ${ }^{4}$ Pathology Department, National Cancer Institute, Cairo University, Cairo, Egypt \\ ${ }^{5}$ Department of Cancer Epidemiology and Biostatistics, National Cancer Institute, Cairo University, Cairo, Egypt \\ Email: `yahia.ismail@nci.cu.edu.eg, amrnci@gmail.com,dr_o_soliman@hotmail.com, neveen.tahoun@nci.cu.edu.eg, \\ allam.rasha@yahoo.com
}

How to cite this paper: Ismail, Y., Kamal, A., Yousof, O., Tahoun, N. and Allam, R. (2019) Primary Bladder Adenocarcinoma: A Five-Year Retrospective Clinicopathologic Study of 42 Cases in Egyptian NCI (2010-14). Journal of Cancer Therapy, 10, 97-116. https://doi.org/10.4236/jct.2019.101008

Received: January 7, 2019

Accepted: January 20, 2019

Published: January 23, 2019

Copyright (๑) 2019 by author(s) and Scientific Research Publishing Inc. This work is licensed under the Creative Commons Attribution International License (CC BY 4.0).

http://creativecommons.org/licenses/by/4.0/

\section{Open Access}

\begin{abstract}
$\underline{\text { Aim: }}$ : Globally, primary adenocarcinoma (AC) accounts for only $0.5 \%-2 \%$ of bladder cancer (BC). Bilharziasis predisposes to $\mathrm{AC}$ in $\sim 10 \%$ of $\mathrm{BC}$ in endemic regions. The aim was to study the clinicopathologic characteristics of this rare entity and define prognostic elements influencing disease-free (DFS) \& overall survival (OS). Patients \& Methods. A retrospective analysis of 42 cases of primary bladder AC presented to the National Cancer Institute of Egypt (NCI-E) during a five-year period (2010-2014), clinicopathologic profiles, management and survival were assessed. Results. The mean age was 55.5 years \pm 9.77 with male predominance. Hematuria, bilharziasis \& urachal type experienced in $88 \%, 35.7 \%$ \& $4.8 \%$, respectively. Radical surgery was employed in $64.3 \%$. Metastatic disease (stageIVB) found in $14.3 \%$, initially. Eleven patients (26.2\%) received palliative chemotherapy for their advanced or metastatic disease, objective response rates (ORR) were $0 \% \& 100 \%$ for those received gemcitabine/platinum \& capecitabine/oxaliplatin, respectively. The 5 -year DFS \& OS rates were $40.7 \% \& 27 \%$, respectively. DFS was significantly enhanced in patients having GII, negative nodes (pN0) and absence of hydronephrosis $(p=0.001,0.011 \& 0.047$, respectively). Presentation with hematuria, $\mathrm{pN} 0$ \& stage II was linked significantly with longer OS ( $p=0.007$, $0.037 \&<0.001$, respectively). Tumour grade $\&$ clinical stage were independent prognostic factors affecting DFS \& OS, respectively on multivariate analysis. Conclusions: Notable reduction in incidence of bladder AC in Egypt is mostly due to decline in bilharzial infection. Tumor grade \& clinical stage are independent prognostic factors for DFS \& OS, respectively. Still no agreement about
\end{abstract}


the role of adjuvant radiotherapy or chemotherapy, however, protocols employed for colorectal cancer seem to be profitable in advanced and metastatic cases. Further larger scale studies are needed to define the hazard factors, molecular characterises and optimal management of this rare type of BC.

\section{Keywords}

Bladder Cancer, Primary, Adenocarcinoma, Bilharziasis, NCI, Egypt

\section{Introduction}

Bladder cancer (BC) positions as the ninth most successive malignant neoplasm globally [1], by far most of patients $(\sim 90 \%)$ have transitional cell carcinoma (TCC) as the main histologic variant in the west, while squamous cell carcinoma (SCC) is more rampant in Africa where there is widespread of Schistosoma hematobium [2]. Primary adenocarcinoma (AC) of the urinary bladder is found in approximately $0.5 \%-2 \%$ of all $\mathrm{BC}$ and ranks as the third most frequent subtype [3] [4] [5]. In Egypt, BC represents one of the major health problems with an overall relative frequency of $18.3 \%$ [6]. Primary AC histology was depicted in $3.8 \%$ of all $\mathrm{BC}$ cases during a twelve-year period pathology cancer registry (2000-2011) of NCI-E [7].

Smoking is still the prominent hazard factor for TCC [8], however, for AC subtype other conceivable risk factors have been depicted e.g. schistosomiasis predisposes to AC in up to one-tenth of all $\mathrm{BC}$ in endemic territories [9] [10] and those with a diagnosis of bladder exstrophy who develop malignant bladder tumours have AC in nearly $90 \%$ of cases [11]. Pathologically, it is derived from the urothelial coating the bladder that demonstrates a pure glandular phenotype which exhibits different microscopic morphologic patterns [5]. Macroscopically, it may present as a papillary, sessile or ulcerating growth that usually originates from the posterior wall but also could be found in other locations in the bladder [11], in contrast to TCC which tends to grow in a multifocal pattern, AC frequently presents as a single lesion [12]. Urachal AC originates from the urachal remnant [13] and represents around one-third of primary bladder AC [5], it is usually found in the bladder vault as a solitary polypoidal lesion and usually grouped together with bladder AC because they have enormous likeness in their features as it exhibits the similar microscopic patterns described in AC with the mucinous subtype being the most frequently experienced [14].

The diagnosis of primary bladder AC should be proposed after meticulous exclusion of secondary AC involving the bladder either by direct intrusion from the surrounding organs or by metastasis from a remote site as they occur more frequent than the former [12] [15], it is usually developed in the sixth or seventh decade of age with male dominance [16] and gross hematuria is the common presenting complaint followed by dysuria and mucusuria [5]. Nearly $33 \%$ of the patients have positive nodal involvement at initial presentation [17] [18]. Pa- 
tients with urachal type tend to present in a younger age than bladder AC with also male predominance, in addition to hematuria they might complain with other symptoms related to its anatomical location like umbilical discharge and lower abdominal pain [5] [13].

Radical cystectomy (RC) accompanied with pelvic lymph node dissection (PLND) is considered the treatment of choice as the highest proportion of the patients have a muscle-invasive disease at presentation, whereas for the minority with superficial disease; transurethral resection of the bladder tumour (TURBT) is often employed [19] [20]. On the other side, the standard surgical approach for urachal tumours is en-bloc resection of the umbilicus, bladder vault and the urachal ligament [21].

The value of radiation therapy whether in adjuvant or neoadjuvant setting is as yet conflicting as AC is generally considered as a radioresistant disease, the five-year survival was previously reported to be less than $20 \%$ in those managed with radiotherapy alone. Two studies showed no survival advantage for preoperative irradiation [22] [23], however, a retrospective study from Egypt exhibited a potential valuable role of adjuvant radiation after RC in diminishing the local recurrence rate with subsequent significant enhancement in DFS [17].

Still, there are no standard chemotherapy guidelines for the treatment of primary AC of the bladder [24] and the usual cisplatin-based protocols that are utilized successfully for TCC demonstrated little advantage on AC [14]. Some researchers recommended that a flouropyrimidine-based regimen should be tried [25] [26]. Applying the same rationale for preoperative chemotherapy in TCC, it might be a sensible option for patients with locally advanced primary $\mathrm{AC}$ as it could help in downstaging the tumour ahead to surgery, eliminating micrometastases, and may diminish regional and distant relapse [27] [28].

In respect to prognosis; the five-year survival rates for AC are commonly low running from $5 \%-33 \%$ [26] [29]. The aim of the current retrospective study is to evaluate the incidence, the clinicopathologic features, the treatment outcome and prognostic elements influencing survival for patients with primary bladder AC in NCI-E.

\section{Patients and Methods}

This is a retrospective cohort study involving patients with a diagnosis of primary AC of the urinary bladder who were treated at the National Cancer Institute of Egypt (NCI-E) over a five-year period; between January, 2010 and December, 2014. There were a total of 3144 cases of BC retrieved from the medical records during the specified period. Eligibility criteria included: age $\geq 18$ years, both genders, definite pathological diagnosis of AC of the urinary bladder whether by cystoscopic biopsy specimen or the whole bladder specimen after radical surgery, final clinical diagnosis of primary AC made after exclusion of secondary (metastatic) AC involving the bladder by: negative colonoscopy and by the use of immunohistochemistry (IHC) - when needed-to exclude exten-

sion or metastasis from other primaries. Exclusion criteria included cases with 
documented clinical diagnosis of metastatic (secondary) AC to the urinary bladder, cases who didn't underwent lower GI endoscopy or suspicious cases that were not subjected to confirmatory IHC. A total of 42 cases out of the 3144 BC cases $(1.3 \%)$ were found to satisfy the inclusion criteria, their archived medical charts and pathology slides were retrieved from the medical records and pathology departments, respectively. The medical charts were thoroughly revised and all the clinicodemographic data were extracted that included: age, sex, residency (urban or rural area), main presenting complaint, date of first diagnosis of a urinary bladder mass and the imaging modality used, date and results of cystoscopy, pathology of cystoscopic biopsy, tumour location within the bladder, tumour size and pattern of macroscopic growth, clinical stage at presentation, date and type of curative surgery, pathological TNM stage for the operable patients, date of relapse and its location, patients presenting with initially metastatic disease and the sites of metastasis, findings of exploration for patients with advanced disease, adjuvant therapy (chemotherapy or radiation therapy) given for radically resected patients, palliative chemotherapy or palliative radiation given for recurrent, advanced or metastatic patients, response to treatment, date and state of last follow up. Patients were retrospectively followed up till the end of May, 2018 (end of data collection).

For patients who underwent curative surgery; RC in male patients included removal of the bladder, prostate gland, seminal vesicles together with perivesical fat and peritoneal coverage, in addition to PLND. Anterior pelvic exenteration (APE) in females included removal of the bladder, perivesical fat, PLND, peritoneal coverage, uterus, ovaries, urethra and anterior vaginal wall as described in [10] [30]. The two patients with urachal carcinoma underwent en-bloc resection of the umbilicus and urachal ligament in addition to partial cystectomy (PC) as described in [21] [31].

The pathologist in charge for this study retrieved and revised the $\mathrm{H} \& \mathrm{E}$ slides for the 42 cases and according to WHO classification for tumours of the urinary bladder; the not-otherwise specified (NOS) subtype shows non-specific glandular growth, the enteric type intimately looks like AC of the colorectum, tumours that show plentiful extracellular mucin with malignant cells lying within the mucin lakes are classified as mucinous adenocarcinoma (MAC), the signet ring adenocarcinoma (SRAC) variant where the cells have high intracellular mucin content that pushes the nucleus to eccentric site of the cell and finally, the mixed pattern demonstrates a mixture of these aforementioned growth patterns [32]. Depth of tumour invasion $(\mathrm{pT})$, nodal status $(\mathrm{pN})$, grade of differentiation and other associated pathology like bilharziasis, cystitis glandularis, cystitis cystica, etc. were documented. Confirmatory IHC was used in one case for a male with pT4a invading the prostate, PSA was negative on the specimen excluding primary prostatic origin in addition to normal serum PSA.

\section{Statistical Methods}

Data were analyzed using IBM SPSS advanced statistics (Statistical Package for 
Social Sciences), version 23 (SPSS Inc., Chicago, IL). Median and range or mean and standard deviation were used as appropriate for numerical data, while qualitative data were described as number and percentage. The relation between qualitative variables was examined by Chi-square (Fisher's exact) test as appropriate. Kaplan-Meier method was done for survival analysis. The log rank test was applied for comparison between two survival curves. Cox regression model was done for multivariate analysis in order to test for independent prognostic effect of statistically significant variables on univariate level with calculating hazard ratio and its 95\% confidence interval. Bonferonni corrections of $\mathrm{p}$ value were done to avoid hyperinflation of type I error which arises from multiple testing. A p-value $\leq 0.05$ was counted as statistically significant. All tests were two tailed. Overall survival (OS) was calculated from the date of diagnosis till the date of death or last follow up. Disease-free survival (DFS) was calculated from the date of curative surgery till the date of local or distant relapse, death or last follows up.

\section{Ethical Issues}

No exposure to any hazards for the patients on this retrospective study. Presentation and gathering of data were absolutely anonymous with maintaining privacy and confidentiality to the maximum possible standards.

\section{Results}

A cohort of 42 cases of primary AC of the urinary bladder, their mean age was 55.5 years \pm 9.77 , median: 57 years (range: 29 - 68), with male predominance (64.3\%) and Male: Female 1.8:1. The majority (69\%) were resident in rural areas in Egypt. Hematuria was the most commonly reported presenting complaint in $88 \%$ of the patients. The method of initial diagnosis for detection of a bladder mass was ultrasound (U/S) of abdomen and pelvis in half of the patients whereas computed tomography (CT) was carried out in the remaining half. Different degrees of hydronephrosis were detected on initial imaging in more than half of the patients (52.4\%). All the patients underwent baseline diagnostic cystoscopy, the most rampant pattern of tumour growth grossly was fungating/exophytic in $83.3 \%$ while the remaining $16.7 \%$ had ulcerative lesions with median tumour size $5 \mathrm{~cm}$ (range: 2 - 20). Posterior wall of the bladder was the most frequent location of the tumours in $57 \%$ followed by the dome in $19 \%$ whereas urachal type was encountered in just two patients (4.8\%). Microscopically, AC-NOS was the pervasive histologic variant in $76.2 \%$ (Figure $1(\mathrm{a})$ ), bilharziasis was found in addition to the bladder tumour in 15 patients (35.7\%, Figure 1(b)) with $93 \%$ of those patients were resident in rural areas, whereas MAC (Figure 1(c)) and SRAC (Figure 1(d)) were found in $14.3 \%$ and $9.5 \%$, respectively, with grade II differentiation depicted in the majority (78.6\%). Clinical diagnosis of muscle-invasive disease (cT2) was detected whether by cystoscopy, imaging or bimanual examination under anaesthesia (EUA) in more than three quarters of 


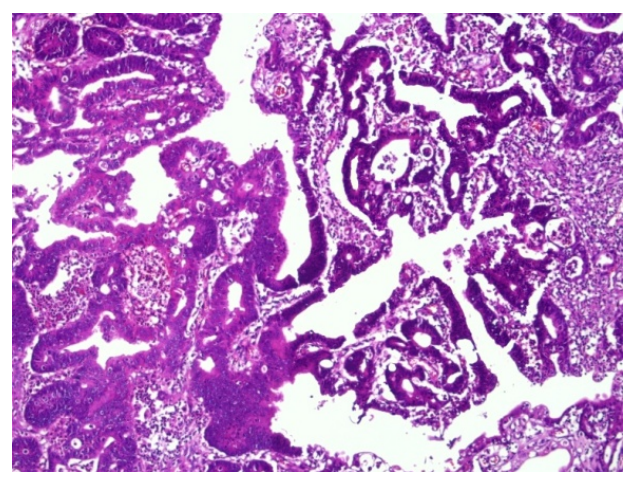

(a)

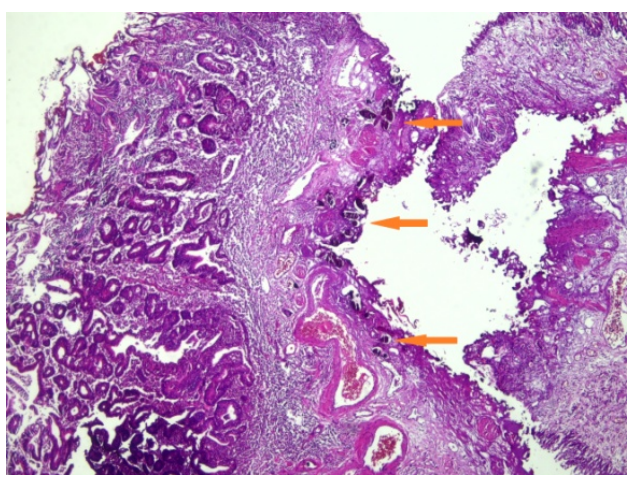

(b)

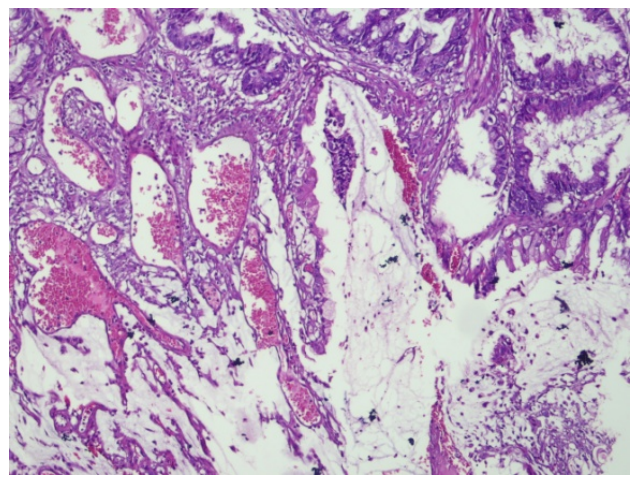

(c)

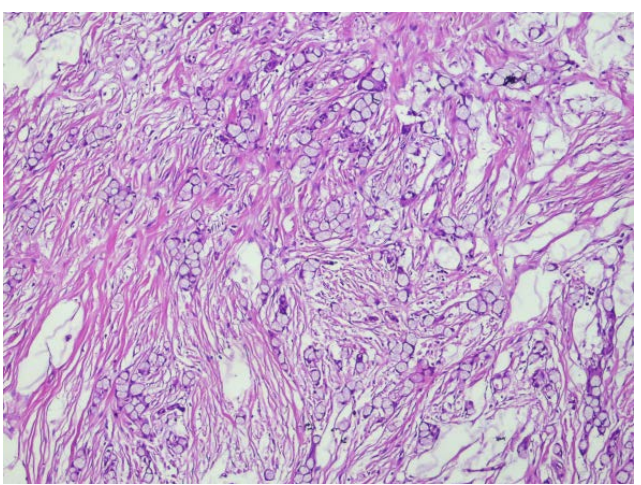

(d)

Figure 1. (a) Primary bladder AC-NOS (H \& E $40 \times 10$ ); (b) Primary bladder AC-NOS associated with bilharziasis (arrows) (H \& E $40 \times 10$ ); (c) Primary bladder mucinous AC (H \& E $40 \times 10)$; (d) Primary bladder signet ring AC (H \& E $40 \times 10)$. 
the patients (76\%), whereas cT3b (gross extravesical extension) in $16.7 \%$. Clinical assessment of lymph node status by imaging and examination revealed clinically negative nodes (cN0) in most of the patients (92\%), consequently; by applying the AJCC staging 8th edition [33]; initial clinical staging for the whole cohort showed that stage II was the most common in almost two thirds of the patients $(66.7 \%)$ followed by clinical stage III and IVA in $16.7 \%$ and $2.3 \%$, respectively, whereas distant metastatic disease at presentation (stage IVB) was anticipated in 6 patients $(14.3 \%)$ with peritoneal nodules being the commonest site of metastasis in almost $67 \%$ of the metastatic patients (Table 1 ). On exploration; 6 patients had inoperable disease due to the presence of peritoneal nodules, fixed pelvic lymph nodes and fixed uterine invasion in 3, 2 \& 1 patients, respectively, whereas curative surgery was successful in 27 patients $(64.3 \%)$ with RC being the most common radical surgery employed in 19 patients followed by APE and PC in 5 and 3 cases, respectively, pT3 was the prevalent stage in 15 patients followed by pT2 \& pT4 in $9 \& 3$ patients, respectively, pathologically positive lymph node was found in only $18.5 \%$ of cases (Table 1).

Table 1. Clincopathologic profiles of 42 cases of primary bladder adenocarcinoma.

\begin{tabular}{|c|c|}
\hline $\begin{array}{c}\text { Age (years): } \\
\text { Mean } \pm \text { SD } \\
\text { Median (range) }\end{array}$ & $\begin{array}{l}55.5 \pm 9.77 \\
57(29-68)\end{array}$ \\
\hline Gender: & $\mathrm{n}(\%)$ \\
\hline Male & $27(64.3)$ \\
\hline Female & $15(35.7)$ \\
\hline Residency: & $\mathrm{n}(\%)$ \\
\hline Urban & $13(31)$ \\
\hline Rural & $29(69)$ \\
\hline Main presenting complaint: & $\mathrm{n}(\%)$ \\
\hline Hematuria & $37(88.1)$ \\
\hline Lower abdominal pain & $2(4.8)$ \\
\hline Dysuria & $1(2.4)$ \\
\hline Urgency & $1(2.4)$ \\
\hline Necroturia & $1(2.4)$ \\
\hline \multicolumn{2}{|l|}{$\begin{array}{l}\text { Methods of initial diagnosis: } \\
\text { Non-Invasive (Imaging): }\end{array}$} \\
\hline US & n (\%) \\
\hline $\mathrm{CT}$ & $21(50)$ \\
\hline Invasive: & $21(50)$ \\
\hline Cystoscopy & $42(100)$ \\
\hline \multicolumn{2}{|l|}{ Tumour size $(\mathrm{cm})$ : } \\
\hline Median (range) & $5(2-20)$ \\
\hline Tumour growth pattern grossly: & $\mathrm{n}(\%)$ \\
\hline Fungating/Exophytic & $35(83.3)$ \\
\hline Ulcerative & $7(16.7)$ \\
\hline Tumour anatomical location: & $\mathrm{n}(\%)$ \\
\hline Posterior wall & $24(57.1)$ \\
\hline Dome & $8(19)$ \\
\hline Anterior wall & $5(11.9)$ \\
\hline Urachal & $2(4.8)$ \\
\hline Left lateral wall & $2(4.8)$ \\
\hline Right Lateral wall & $1(2.4)$ \\
\hline
\end{tabular}




\section{Continued}

\begin{tabular}{|c|c|}
\hline Pathological subtype: & $\mathrm{n}(\%)$ \\
\hline $\mathrm{AC}(\mathrm{NOS})$ & $32(76.2)$ \\
\hline MAC & $6(14.3)$ \\
\hline SRAC & $4(9.5)$ \\
\hline Tumour grade: & $\mathrm{n}(\%)$ \\
\hline II & $33(78.6)$ \\
\hline III & $9(21.4)$ \\
\hline Associated bilharziasis: & $\mathrm{n}(\%)$ \\
\hline Negative & $27(64.3)$ \\
\hline Positive & $15(35.7)$ \\
\hline Intitial clinical stage $e^{*}$ : & $\mathrm{n}(\%)$ \\
\hline Stage II & $28(66.7)$ \\
\hline Stage III & $7(16.7)$ \\
\hline Stage IVA & $1(2.3)$ \\
\hline Stage IVB (distant metastasis) & $6(14.3)$ \\
\hline Curative radical Surgery $(n=27)$ : & $\mathrm{n}(\%)$ \\
\hline $\mathrm{RC}$ & $19(70.4)$ \\
\hline APE & $5(18.5)$ \\
\hline PC & $3(11.1)$ \\
\hline Pathological tumour $(\mathrm{p} T)$ stage $(\mathrm{n}=27)$ : & $\mathrm{n}(\%)$ \\
\hline pT2 & $9(33.3)$ \\
\hline pT3a & $5(18.5)$ \\
\hline pT3b & $10(37.1)$ \\
\hline pT4 & $3(11.1)$ \\
\hline Pathological nodal involvement $(n=27)$ : & $\mathrm{n}(\%)$ \\
\hline Negative & $22(81.5)$ \\
\hline Positive & $5(18.5)$ \\
\hline
\end{tabular}

*according to TNM 8th edition [33], AC: adenocarcinoma, APE: anterior pelvic exenteration, MAC: mucinous adenocarcinoma, NOS: not-otherwise specified, PC: partial cystectomy, RC: radical cystectomy, SD: standard deviation, SRAC: signet ring adenocarcinoma.

In respect to chemotherapy employed; adjuvant treatment with 4 cycles of gemcitabine and cisplatin was offered to just one case (3.7\%) with pT3bN0M0 (stage IIIA). Palliative chemotherapy was given to 11 (26.2\%) patients (recurrent, locally advanced and metastatic) with median number of cycles was 6 (range: 1 - 8), gemcitabine and platinum regimen was employed in all cases except one patient who received flouropyrimidine-based regimen (Capecitabine and Oxaliplatin/XELOX). The objective response rate (ORR) in the ten patients who received platinum (Cisplatin in 5 patients and Carboplatin in the other 5) was $0 \%$ as 9 cases (90\%) showed disease progression (PD) and one patient (10\%) had stable disease (SD) according to response evaluation criteria in solid tumours (RECIST v1.1) [34], regarding the remaining patient who received XELOX; partial response (PR) was the clinical outcome after 6 cycles. Concerning radiation therapy; one patient (3.7\%) with pT2bN0M0 (stage II) received adjuvant radiation whereas, palliative irradiation was employed in 9 patients (21.4\%) for control of persistent hematuria, bone metastases, brain metastases and pelvic recurrence in 5, 2, 1 and 1 patients, respectively.

Survival: At the end of follow up period (median: 13 months, range: 1 - 82), 
twenty two patients were dead. The median DFS and OS were 17.8 \& 20.2 months, respectively. The cumulative DFS and OS at 1, 3 \& 5 years were $58.7 \%, 40.7 \%$ \& $40.7 \%$ and $69 \%, 35.7 \%$ \& 26.8\%, respectively (Figure 2(a) \& Figure 2(b)). Patients who had negative lymph nodes pathologically, GII tumours and absence of hydronephrosis on imaging had significantly longer DFS contrasted to those with positive nodes $(p=0.011)$, GIII tumours $(p=0.001)$ and hydronephrosis $(p$ $=0.047$ ), respectively (Table 2 , Figures $3(\mathrm{a})-(\mathrm{c})$ ). Cases presented with hematuria, initial clinical stage II and pathologically negative nodes had significantly longer OS compared to those presented with other main complaints $(p=0.007)$, stage III \& IV disease $(p<0.001)$ and positive nodes $(p=0.037)$, respectively (Table 3, Figures 4(a)-(c)). Age, sex, residency, type of surgery, pathological subtype, grade, presence of bilharziasis, pattern of tumour growth, tumour size and location did not have significant impact on DFS \& OS (Table 2 \& Table 3 ). On multivariate analysis, the independent prognostic factor that affected DFS was tumour grade with cases having GIII showed worse DFS compared to GII ( $p$ $=0.006$ ) with HR 7.81 (CI 1.79 - 33.98), whereas the only independent variable affected OS was the clinical stage as patients with stage III \& IV showed worse survival than stage II ( $p=0.001)$ with HR 4.67 (CI $1.90-11.47)$.

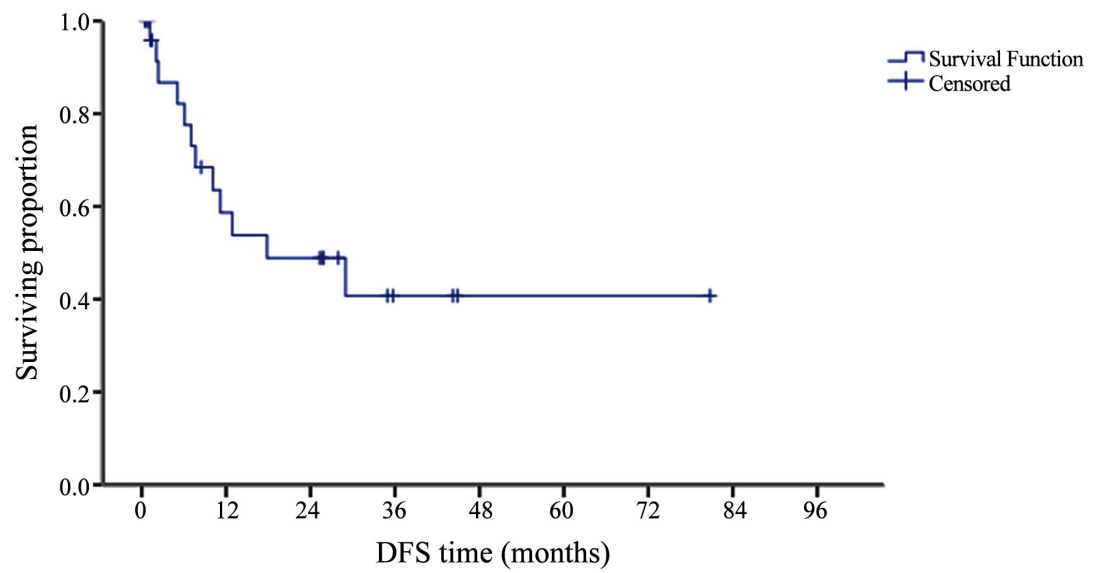

(a)

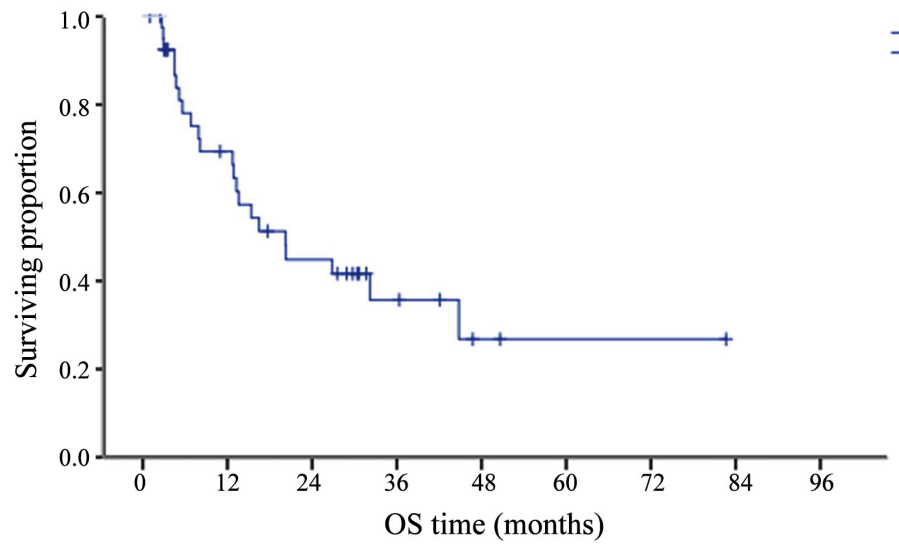

$\neg$ Survival Function - Censored

(b)

Figure 2. (a) DFS for the whole cohort; (b) OS for the whole cohort. 


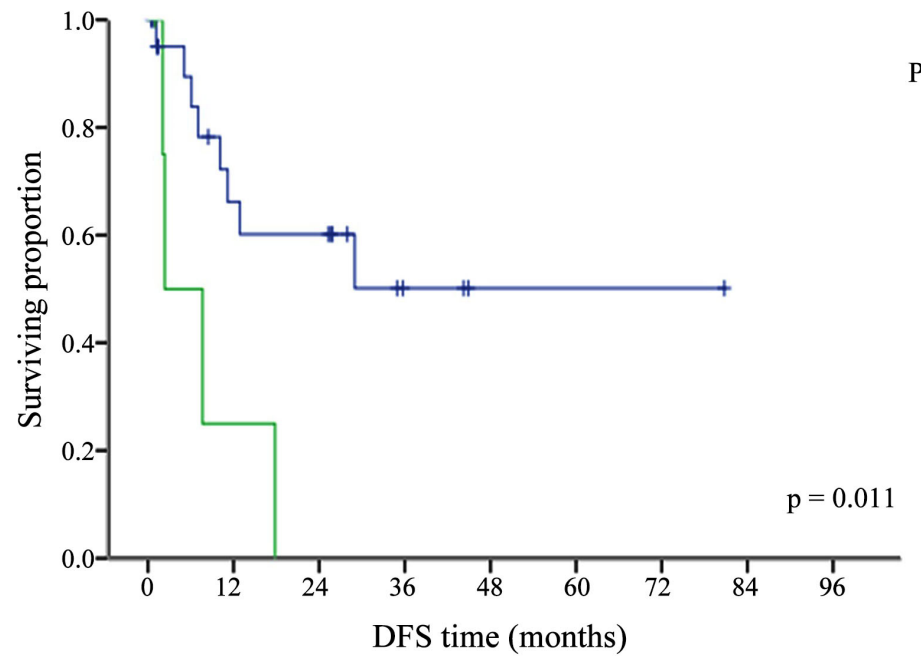

Pathological nodal stage $(\mathrm{pN})$

$\neg \mathrm{pNO}$

$\neg \mathrm{pN}+\mathrm{ve}$

+ pN0-censored

$+\mathrm{pN}+$ ve-censored

(a)

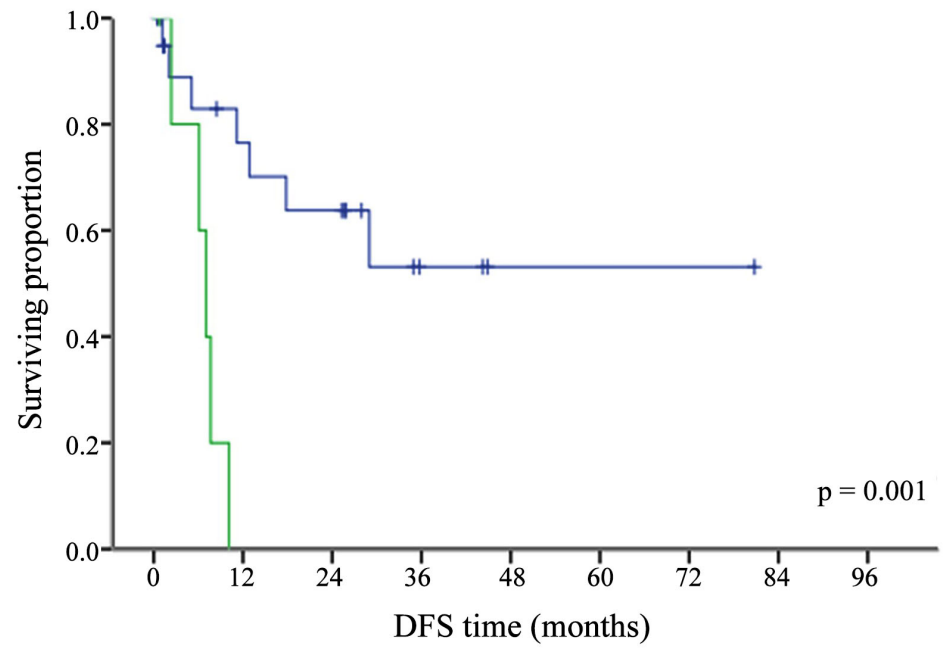

Tumor grade $(\mathrm{G})$

$\neg$ GII

ᄀGIII

+ GII-censored

+ GIII-censored

(b)

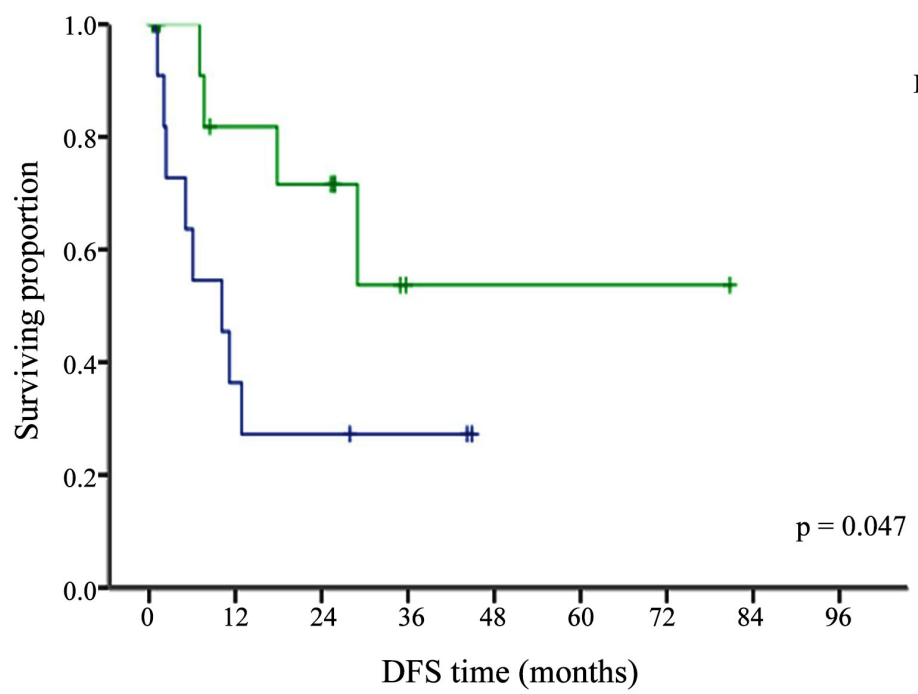

Hydronephosis on initial imaging

$\neg$ present

$\neg$ absent

+present-censored

+ absent-censored

(c)

Figure 3. (a) DFS according to pathological nodal status; (b) DFS according to tuomur grade; (c) DFS according to hydronephrosis status. 


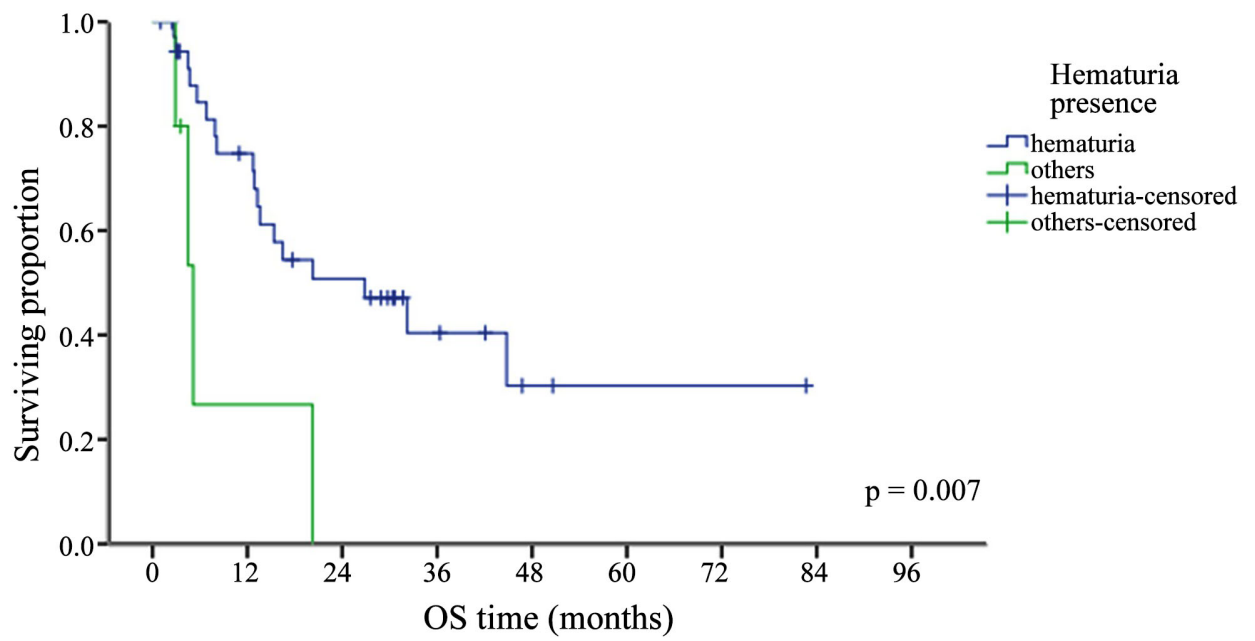

(a)

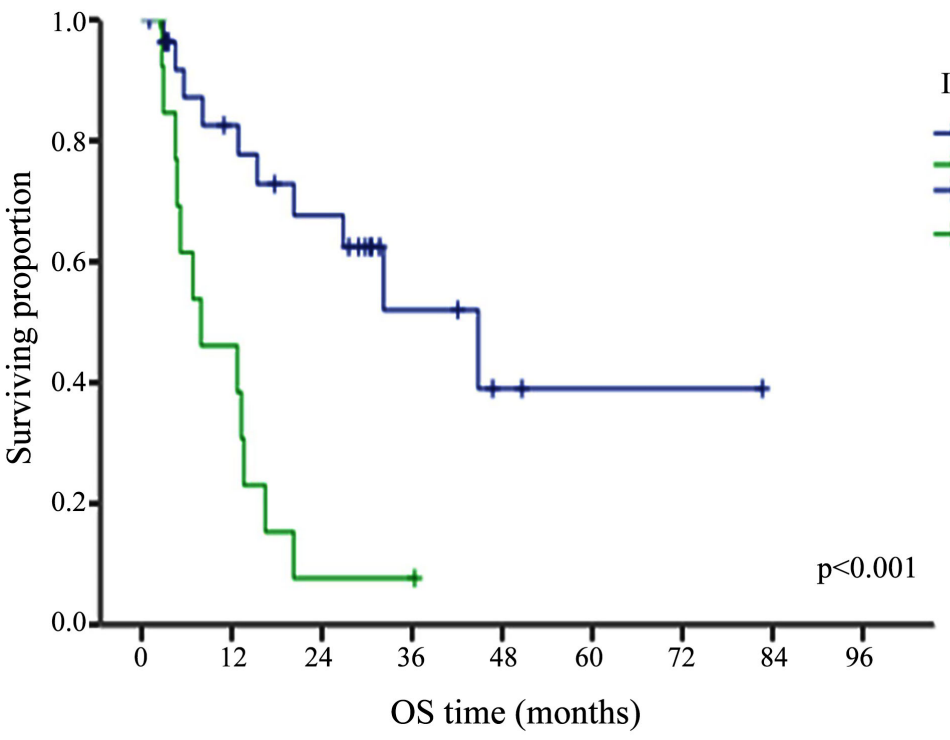

Initial clinical staging

$\neg$ clnical stage II

I clnical stage III \& IV

+ clnical stage II-censored

+ clnical stage III \& IV.

(b)

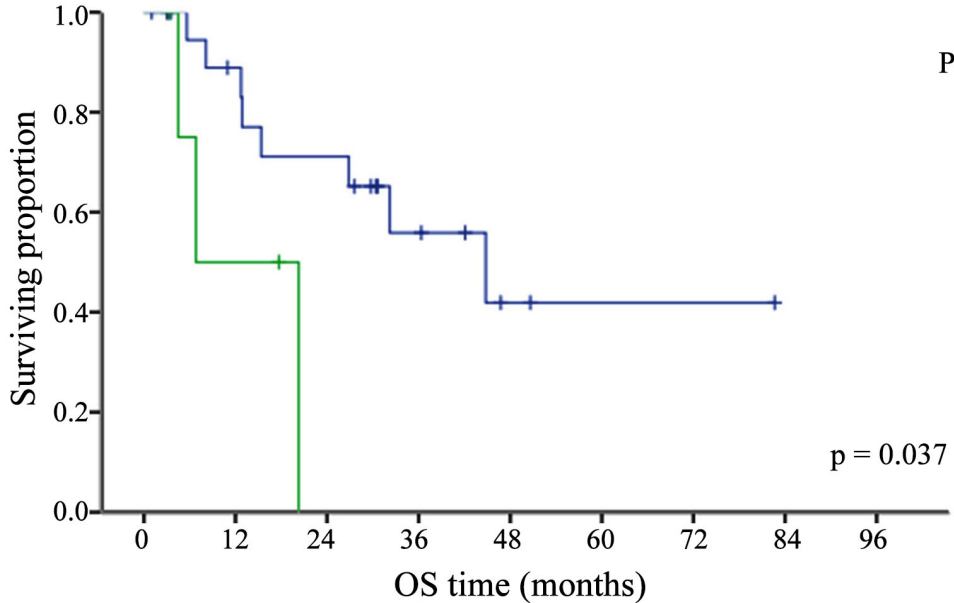

Pathological nodal stage $(\mathrm{pN})$

$\neg$ pN0

+ pN0-censored

+ pN+-censored

(c)

Figure 4. (a) OS according to presentation with hematuria; (b) OS according to initial clinical stage; (c) OS according to pathological nodal status. 
Table 2. Disease-free survival (DFS) in relation to clinicopathologic characteristics.

\begin{tabular}{|c|c|c|c|c|c|c|c|}
\hline Characteristic & $\mathrm{N}$ & $\mathrm{N}$ of events & $\begin{array}{l}\text { Cumulative DFS } \\
\text { at } 12 \mathrm{~ms}(\%)\end{array}$ & $\begin{array}{l}\text { Cumulative DFS } \\
\text { at } 36 \mathrm{~ms}(\%)\end{array}$ & $\begin{array}{c}\text { Cumulative DFS at } \\
60 \mathrm{~ms}(\%)\end{array}$ & $\begin{array}{c}\text { Median DFS } \\
\text { estimate (ms) }\end{array}$ & $P$-value \\
\hline Whole group & 27 & 12 & 58.7 & 40.7 & 40.7 & 17.83 & NA \\
\hline \multicolumn{8}{|l|}{ Gender: } \\
\hline Male & 18 & 7 & 63.5 & 41.7 & NR & 28.98 & 0.569 \\
\hline Female & 9 & 5 & 50.8 & 38.1 & 38.1 & 17.83 & \\
\hline \multicolumn{8}{|l|}{ Age Categories: } \\
\hline$\leq 50$ & 9 & 3 & 85.7 & 51.4 & 51.4 & NR & 0.327 \\
\hline$>50$ & 18 & 9 & 46.9 & 31.3 & NR & 11.18 & \\
\hline$\leq 60$ & 17 & 8 & 60.6 & 32.5 & 32.5 & 17.83 & \\
\hline$>60$ & 10 & 4 & 55.6 & 55.6 & NR & NR & 0.659 \\
\hline \multicolumn{8}{|l|}{ Residence: } \\
\hline Rural & 19 & 7 & 52.3 & 52.3 & 52.3 & NR & 0.727 \\
\hline Urban & 8 & 5 & 71.4 & NR & NR & 17.83 & \\
\hline \multicolumn{8}{|l|}{ Curative Sx: } \\
\hline $\mathrm{RC}+\mathrm{PC}$ & 22 & 10 & 58.6 & 34.2 & NR & 17.83 & 0.509 \\
\hline APE & 5 & 2 & 60.0 & 60.0 & 60.0 & NA & \\
\hline \multicolumn{8}{|l|}{ Main complaint: } \\
\hline Hematuria & 25 & 11 & 61.4 & 42.6 & 42.6 & 28.98 & * \\
\hline Others & 2 & 1 & NR & NR & NR & 2.37 & \\
\hline \multicolumn{8}{|l|}{ Tumour Growth: } \\
\hline \multicolumn{8}{|l|}{ Fungating } \\
\hline \multicolumn{8}{|l|}{ Ulcerative } \\
\hline \multicolumn{8}{|l|}{ Tumour Size $(\mathrm{cm})$} \\
\hline$\leq 5$ & 21 & 12 & 50.2 & 29.3 & 29.3 & 18.0 & 0.054 \\
\hline$>5$ & 6 & 0 & 100 & NR & NR & NR & \\
\hline \multicolumn{8}{|c|}{ Tumour Location: } \\
\hline Posterior wall & 16 & 7 & 60.6 & 41.5 & 41.5 & 28.98 & 0.818 \\
\hline Others & 11 & 5 & 56.3 & 45.0 & NR & 12.86 & \\
\hline \multicolumn{8}{|l|}{ Clinical stage $^{\#}$} \\
\hline II & 24 & 10 & 63.1 & 41.3 & NR & 28.98 & * \\
\hline III \& IV & 3 & 2 & 33.3 & NR & NR & 10.13 & \\
\hline \multicolumn{8}{|l|}{ pT: } \\
\hline pT2 & 9 & 2 & 85.7 & 57.1 & 57.1 & NR & 0.126 \\
\hline pT3 \& 4 & 18 & 10 & 46.9 & 33.5 & NR & 11.18 & \\
\hline \multicolumn{8}{|l|}{ pN: } \\
\hline Negative & 22 & 8 & 66.2 & 50.2 & 50.2 & NR & 0.011 \\
\hline Positive & 5 & 4 & 25.0 & NR & NR & 2.37 & \\
\hline \multicolumn{8}{|l|}{ Hydronephrosis: } \\
\hline Present & 13 & 8 & 36.4 & 27.3 & NR & 10.13 & 0.047 \\
\hline Absent & 14 & 4 & 81.8 & 53.7 & 53.7 & NR & \\
\hline \multicolumn{8}{|l|}{ Pathology: } \\
\hline AC (NOS) & 21 & 9 & 64.6 & 41.3 & NR & 28.98 & 0.720 \\
\hline MAC \& SRAC & 6 & 3 & 40.0 & 40.0 & 40.0 & 10.13 & \\
\hline \multicolumn{8}{|l|}{ Tumour grade: } \\
\hline II & 21 & 7 & 76.5 & 53.1 & 53.1 & NR & 0.001 \\
\hline III & 6 & 5 & NR & NR & NR & 7.04 & \\
\hline \multicolumn{8}{|l|}{ Bilharziasis: } \\
\hline Yes & 13 & 6 & 36.0 & 36 & 36 & 10.13 & 0.272 \\
\hline No & 14 & 6 & 75.0 & 43.8 & NR & 28.98 & \\
\hline
\end{tabular}

\#according to TNM 8th edition [33], *p value not applicaple, AC: adenocarcinoma, APE: anterior pelvic exenteration, MAC: mucinous adenocarcinoma, ms: month, n: number, NA: not applicable, NOS: not-otherwise specified, PC: partial cystectomy, pN: pathological nodal status, pT: pathological tumor stage, RC: radical cystectomy, SRAC: signet ring adenocarcinoma, Sx: surgery. 
Table 3. Overall survival (OS) in relation to clinicopathologic characteristics.

\begin{tabular}{|c|c|c|c|c|c|c|c|}
\hline Characteristic & $\mathrm{N}$ & $\mathrm{N}$ of events & $\begin{array}{c}\text { Cumulative OS at } \\
12 \mathrm{~ms}(\%)\end{array}$ & $\begin{array}{c}\text { Cumulative OS at } \\
36 \mathrm{~ms}(\%)\end{array}$ & $\begin{array}{c}\text { Cumulative OS at } \\
60 \mathrm{~ms}(\%)\end{array}$ & $\begin{array}{c}\text { Median OS } \\
\text { estimate (ms) }\end{array}$ & $P$-value \\
\hline Whole cohort: & 42 & 22 & 69.4 & 35.7 & 26.8 & 20.23 & NA \\
\hline \multicolumn{8}{|l|}{ Gender: } \\
\hline Male & 27 & 13 & 74.1 & 39.1 & NR & 26.84 & 0.454 \\
\hline Female & 15 & 9 & 61.5 & 30.8 & 30.8 & 12.86 & \\
\hline \multicolumn{8}{|l|}{ Age categories: } \\
\hline$\leq 50$ & 10 & 3 & 100 & 51.4 & 51.4 & NR & 0.085 \\
\hline$>50$ & 32 & 19 & 60.2 & 28.2 & NR & 13.29 & \\
\hline$\leq 60$ & 24 & 13 & 69.5 & 31.3 & 20.9 & 20.23 & \\
\hline$>60$ & 18 & 9 & 88.1 & 44.1 & NR & 26.84 & 0.657 \\
\hline \multicolumn{8}{|l|}{ Residence: } \\
\hline Rural & 29 & 14 & 67.7 & 33.2 & 33.2 & 20.23 & 0.662 \\
\hline Urban & 13 & 8 & 72.7 & 36.4 & NR & 15.36 & \\
\hline \multicolumn{8}{|l|}{ Main complaint: } \\
\hline Hematuria & 37 & 18 & 74.8 & 40.4 & 30.3 & 26.84 & 0.007 \\
\hline Others & 5 & 4 & 26.7 & NR & NR & 5.16 & \\
\hline \multicolumn{8}{|l|}{ Tumor growth: } \\
\hline Fungating & 35 & 21 & 70.9 & 29.1 & 19.4 & 16.48 & 0.108 \\
\hline Ulcerative & 7 & 1 & 80 & 80 & NR & NR & \\
\hline \multicolumn{8}{|l|}{ Tumor Size $(\mathrm{cm})$} \\
\hline$\leq 5$ & 24 & 14 & 67.3 & 36.2 & 24.2 & 16.48 & 0.694 \\
\hline$>5$ & 18 & 8 & 72.4 & 24.8 & NR & 20.23 & \\
\hline \multicolumn{8}{|l|}{ Tumor location: } \\
\hline Posterior wall & 25 & 12 & 70.4 & 51.2 & 34.1 & 44.80 & 0.296 \\
\hline Other sites & 17 & 10 & 66.7 & 16.7 & NR & 13.58 & \\
\hline \multicolumn{8}{|l|}{ Tumor grade: } \\
\hline II & 33 & 16 & 67.7 & 41.0 & 30.7 & 26.84 & 0.279 \\
\hline III & 9 & 6 & 75.0 & NR & NR & 12.86 & \\
\hline \multicolumn{8}{|l|}{ Hydronephrosis: } \\
\hline Present & 22 & 15 & 58.8 & 17.8 & NR & 13.29 & 0.065 \\
\hline Absent & 20 & 7 & 81.9 & 60.6 & 30.3 & 44.80 & \\
\hline \multicolumn{8}{|l|}{ Pathology: } \\
\hline AC (NOS) & 32 & 16 & 70.4 & 38.0 & NR & 20.29 & 0.600 \\
\hline MAC \& SRAC & 10 & 6 & 66.7 & 33.3 & 33.3 & 13.28 & \\
\hline \multicolumn{8}{|l|}{ Bilharziasis: } \\
\hline Present & 15 & 8 & 59.1 & 26.3 & 26.3 & 12.73 & 0.623 \\
\hline Absent & 27 & 14 & 74.7 & 43.2 & NR & 20.23 & \\
\hline \multicolumn{8}{|l|}{ Clinical stage $^{\#}$} \\
\hline Stage II & 28 & 10 & 82.5 & 52.0 & 39.0 & 44.80 & $<0.001$ \\
\hline Stage III \& IV & 14 & 12 & 46.2 & 7.7 & NR & 7.93 & \\
\hline \multicolumn{8}{|c|}{ Curative $S \mathbf{x}(\mathrm{n}=27)$} \\
\hline $\mathrm{RC}+\mathrm{PC}$ & 22 & 9 & 82.4 & 44.6 & NR & 32.24 & 0.735 \\
\hline APE & 5 & 2 & 80 & 60 & 60 & NR & \\
\hline \multicolumn{8}{|l|}{$\mathrm{pT}(\mathrm{n}=27)$} \\
\hline pT2 & 9 & 2 & 100 & 75 & NR & 44.8 & 0.116 \\
\hline pT3 \& 4 & 18 & 9 & 73.3 & 38.1 & NR & 20.3 & \\
\hline \multicolumn{8}{|l|}{$\mathrm{pN}(\mathrm{n}=27)$} \\
\hline Negative & 22 & 8 & 88.9 & 55.9 & 41.9 & 44.80 & 0.037 \\
\hline Positive & 5 & 3 & 50.0 & NR & NR & 6.84 & \\
\hline
\end{tabular}

\#according to TNM 8th edition [33], AC: adenocarcinoma, APE: anterior pelvic exenteration, MAC: mucinous adenocarcinoma, n: number, NA: not applicable, NOS: not-otherwise specified, NR: not reached, PC: partial cystectomy, pN: pathological nodes, pT: pathological tumor stage, RC: radical cystectomy, SRAC: signet ring adenocarcinoma, Sx: surgery. 


\section{Discussion}

In the current retrospective series of 42 cases of primary bladder $\mathrm{AC}$, the mean age was 55.5 years which is almost identical to Korkes et al. (55.8) [35], relatively close to Grignon et al. (58.3) [5] and Zaghloul et al. (50.4) [17] and almost 10 years older than in El-Mekresh et al. (46.3) [9], the latter finding could be referred to the discrepancy of the number of patients and the study duration. There was a male predominance (64\%) with M:F ratio 1.8:1, concurring with previous reports from Egypt [9] [17] and also western reports [5] [36], whereas all the patients (100\%) were males in [35]. This sex predilection could be interpreted by that males might be exposed to the hazard factors for developing bladder AC more than females e.g. to bilharziasis in endemic areas through farming work or to chronic irritation by infection and other risk factors in other areas.

The worldwide incidence of primary bladder AC reported in literature is $0.5 \%$ - $2 \%$ of all BC [3] [4] [5], however, the incidence is higher in areas known with bilharziasis endemicity, ranging from 5\% - 11.4\% [30] [37] [38] as documented by old series from Egypt e.g. El-Bolkainy et al. reported an incidence of $8.1 \%$ in a series of 229 BC cases developed on top of bilharziasis over the period (1967-1970) [39], also El-Mekresh et al. found an incidence of $9.9 \%$ as they demonstrated 185 cases (82\% had bilharziasis) of primary AC out of $1870 \mathrm{BC}$ patients post RC over a 25 -year period (1970-1995) [9], these reports could be an evidence confirming that bilharziasis is one of the well documented predisposing factors for the development of bladder AC through inducing metaplastic changes of potentially unsteady urothelium as described in [40] [41], however, the incidence of bladder AC showed progressive decline in subsequent published Egyptian series e.g. it was $5.2 \%$ in the work of Zaghloul et al. as they described 192 bladder AC out of 3659 patients who underwent cystectomy over a ten-year period (1994-2003) [17], it was 3.8\% of all BC cases presented to NCI-E (which is the largest tertiary cancer centre in Egypt) over a twelve- year period (2000-2011) [7], in the present retrospective series, we depicted 42 cases of primary bladder AC out of a total of $3144 \mathrm{BC}$ cases presented to NCI-E during the studied five-year period (2010-14) representing an incidence of $1.3 \%$ which corresponds to the range of the reported global incidence, this downward trend might be strongly explained by the continuing decline in bilharzial infection in Egypt during the last three decades compared to the era of 1970s and 1980s owing to mass governmental struggling and the wide spread availability of anti-bilharzial treatment in the primary health care facilities distributed all over the rural areas of Egypt. In contrast; the old series coming from the States reported an incidence of bladder AC that in congruence with the worldwide incidence; $1.6 \%$ during the period (1973-77) in the work of Young et al. [42], Grignon et al. described only 72 cases through a so long 40 -year period (1948-1987) [5], this discrepancy in incidence between old Egyptian and western series mostly due to the high prevalence of infection with shistosoma haemato- 
bium in rural Egypt compared to the west.

In the present study, the $1 \& 5$-year OS were $69 \% \& 26.8 \%$, respectively, which corresponds to the previously reported ranges; $23.1 \%-87.5 \%$ \& $5 \%-33 \%$, respectively described in [26] [29], whereas the current 5-year DFS rate was $40.7 \%$ close to $46 \%$ in [17] while it was $55 \%$ in [9]. As regard the relation of different prognostic elements to survival parameters; hematuria which was the chief presenting complaint by the highest percent of patients in the present study (88\%), that is similar to previous reports in [5] [35] [43], our outcome showed that those patients had significantly longer OS contrasted to the cases presented with other main complaints ( $p=0.007)$, the authors could owe this finding to the fact that gross hematuria is a pressing caution sign that makes patients seeking medical counselling urgently with consequent diagnosis of the tumour at an early stage. Patients presented with preoperative hydronephrosis in the current series exhibited significantly shorter DFS compared to others without ( $p=$ 0.047), a finding which exactly concurring with the work of Bartsch et al. [44] and Lin et al. [45] as hydronephrosis was significantly related to higher $\mathrm{T}$ stages in their works which included patients with only urothelial carcinoma contrasting to our study of bladder AC only. High grade (III) tumours demonstrated significantly shorter DFS ( $p=0.001$ ), similar results found in [9] [17], moreover, it was an independent prognostic factor predicting worse DFS on multivariate analysis.

Clinical stage at presentation demonstrated a significant effect on OS in the current study; moreover, it was an independent prognostic factor for OS on multivariate analysis, comparable outcomes were reported in [5]. Up to $33 \%$ of bladder AC patients have nodal involvement at the moment of presentation [17] [18], in the current study; positive lymph node metastasis was found in $18.5 \%$ of the resected patients which is coinciding with El-Bolkainy et al. (19\%) [39], those patients experienced significantly shorter DFS \& OS compared to node negative cases ( $p=0.011 \& 0.037$, respectively) similar to the reports of [9] [17].

Fungating/exophytic growth pattern was the most widely recognized finding on initial cystoscopy in $83.3 \%$ of the patients in the current series, with posterior wall being the most frequent location of the bladder tumours in $57 \%$, typically as reported by [11] [46] and also similar to [39] when they described features of BC developed on bilharziasis in Egypt. AC-NOS was the pervasive histological subtype in the series in hand (76.2\%), these results are in congruence with those of [5] [17], though the MAC histology was the overwhelming type in [9]. We couldn't delineate significant effect of AC subtypes on the DFS \& OS in consistence with [5] and in contradiction to [17] who reported that the SRAC subtype had significantly worst 5-year DFS compared to other histological subtypes ( $p=$ $0.00001)$, the latter result is concurring with the work of Holmang et al. who reported that SRAC is frequently diagnosed in advanced stage and has a bleak survival rate [47]. The authors might explain this opposing finding by the relatively small number of the whole cohort in the current study compared to [17] (42 vs. 192 , respectively) and also the small numbers in the subgroups. 
The role of adjuvant radiation for bladder $\mathrm{AC}$ was previously raised in a retrospective study from Egypt where post operative radiotherapy enhanced the 5 -year DFS and the local control rates compared to cystectomy alone ( $p=0.002$ $\& 0.00001$, respectively) specially in locally advanced disease (pT3, pT4a) [17], however, in the present study; despite $55.6 \%$ and $17 \%$ of the patients had pT3 and pT4, respectively; radiotherapy was mostly used for palliative intent with adjuvant radiotherapy employed in just a single patient (3.7\%) who had pT2b, N0 stage, subsequently we couldn't derive any conclusions about the impact of adjuvant radiation on the outcome. The authors refer this to the existing absence of level 1 evidence supporting the use of adjuvant radiation in this rare $\mathrm{BC}$ subtype and also to the prevailed belief between oncologists that this subtype is mostly radioresistant as reported in other studies [22] [23].

The role of chemotherapy described for this rare type of $\mathrm{BC}$ in the neoadjuvant, adjuvant or metastatic setting still represents an area of great debate and needs continuing intense research. In the current series, we depicted ORR $0 \%$ for the $10(23.8 \%)$ patients with recurrent, advanced or metastatic disease who received gemcitabine/platinum regimen used for treating TCC whereas it was $100 \%$ for the single patient who received XELOX regimen as for treating adenocarcinoma of primary colorectal origin. These findings reflect the aforementioned lack of consensus between medical oncologist regarding the optimal type of chemotherapy for this tumour and that most of oncologist opt to use conventional protocols employed in combating the most frequent type of bladder cancer (TCC) rather than to be pathology-directed. Similar findings were reported by Siefker-Radtke et al. who retrospectively analysed 42 cases of urachal carcinoma at M.D. Anderson Cancer Centre during a 17-year period (1985-2001), they found ORR $33 \%$ in patients with metastatic disease who received palliative chemotherapy containing 5-fluorouracil, so they concluded that regimens used for primary colonic AC might be more advantageous for this tumour than conventional regimens given for TCC, consequently; they stated that the GFLP [gemcitabine, 5-fluorouracil, leucovorin and cisplatin] is their preferred initial protocol for metastatic urachal AC [26]. Another study supporting these data done by Yanagihara et al. who retrospectively evaluated five patients with metastatic urachal cancer received mFOLFOX6 regimen, the ORR was $40 \%$ and concluded that this protocol appears to be successful for the management of patients with metastatic urachal cancer [48], also in consistence with the latter conclusion; Fan \& Yang reported long lasting response to mFOLFOX6 and capecitabine for a patient with metastatic primary bladder nonurachal AC [49].

\section{Conclusion}

The authors admit the limitations of retrospective studies, but retrospectivity is the most appropriate way for studying such a rare type of bladder cancer $(0.5 \%$ 2\%). A notable continuing decrease of the incidence of bladder AC in Egypt is mostly due to the dramatic decrease in bilharzial infection, the incidence in this series $(1.3 \%)$ is within the worldwide range. The reported 5-year DFS \& OS in 
the current work are in congruence with previous Egyptian and western series. The tumour grade and the clinical stage at presentation were independent prognostic factors for DFS \& OS, respectively. Radical surgery is the optimal treatment for locoregional disease but still no consensus about the role of adjuvant radiotherapy or chemotherapy. Antineoplastic protocols used for adenocarcinoma of colorectal cancer seem to be profitable in advanced and metastatic cases. Further larger scale studies are needed to define other risk factors, molecular characterises and optimal management of this unique type of bladder cancer.

\section{Conflicts of Interest}

The authors declare no conflicts of interest regarding the publication of this paper.

\section{References}

[1] Ferlay, J., Soerjomataram, I., Dikshit, R., Eser, S., Mathers, C., Parkin, D.M., et al. (2015) Cancer Incidence and Mortality Worldwide: Sources, Methods and Major Patterns in GLOBOCAN 2012. International Journal of Cancer, 136, E359-E386. https://doi.org/10.1002/ijc.29210

[2] Mostafa, M.H., Sheweita, S.A. and O’Connor, P.J. (1999) Relationship between Schistosomiasis and Bladder Cancer. Clinical Microbiology Review, 12, 97-111. https://doi.org/10.1128/CMR.12.1.97

[3] Thomas, D.C., Ward, A.M. and Williams, J.L. (1971) A Study of 52 Cases of Adenocarcinoma of the Bladder. British Journal of Urology, 43, 4-15. https://doi.org/10.1111/j.1464-410X.1971.tb04925.x

[4] Burnett, A.L., Epstein, J.I. and Marshall, F.F. (1991) Adenocarcinoma of Urinary Bladder: Classification and Management. Urology, 37, 315-321. https://doi.org/10.1016/0090-4295(91)80256-7

[5] Grignon, D.J., Ro, J.Y., Ayala, A.G., Johnson, D.E. and Ordóñez, N.G. (1991) Primary Adenocarcinoma of the Urinary Bladder. A Clinicopathologic Analysis of 72 Cases. Cancer, 67, 2165-2172. https://doi.org/10.1002/1097-0142(19910415)67:8<2165::AID-CNCR2820670827>3. $\underline{0 . \mathrm{CO} ; 2-\mathrm{M}}$

[6] El-Bolkainy, M.N. (2016) Epidemiology of Cancer. In: El-Bolkainy, M.N., Nouh, M.A., Gouda, I., El-Bolkainy, T.N. and Badawy, O.M., (Eds.), Pathology of Cancer, 5th Edition, NCI-E Publication, Cairo, Egypt, 15-31.

[7] Khorshed, E. (2016) Urinary System Tumors. In: Mokhtar, N., Asmaa, S., Badawy, O., Khorshed, E., Mohamed, G., Ibrahim, M., et al., Eds., Cancer Pathology Registry 2000-2011, Cairo Press, Cairo, Egypt, 60-74.

[8] Burger, M., Catto, J.W., Dalbagni, G., Grossman, H.B., Herr, H., Karakiewicz, P., et al. (2013) Epidemiology and Risk Factors of Urothelial Bladder Cancer. European Urology, 63, 234-241. https://doi.org/10.1016/j.eururo.2012.07.033

[9] El-Mekresh, M.M., El-Baz, M.A., Abol-Enein, H. and Ghoneim, M.A. (1998) Primary Adenocarcinoma of the Urinary Bladder: A Report of 185 Cases. British Journal of Urology, 82, 206-212. https://doi.org/10.1046/j.1464-410X.1998.00718.x

[10] Ghoneim, M.A., Abdel-Latif, M., El-Mekresh, M., Abol-Enein, H., Mosbah, A., Ashamallah, A. and ElBaz, M.A. (2008) Radical Cystectomy for Carcinoma of the 
Bladder: 2,720 Consecutive Cases 5 Years Later. Journal of Urology, 180, 121-127. https://doi.org/10.1016/j.juro.2008.03.024

[11] Epstein, J.I., Amin, M.B. and Reuter, V.E. (2010) Glandular Lesions. In: Epstein, J.I., Amin, M.B. and Reuter, V.E., Eds., Biopsy Interpretation of the Bladder, 2nd Edition, Lippincott Williams \& Wilkins, Philadelphia, PA, 180-213.

[12] Melicow, M.M. (1955) Tumors of the Urinary Bladder: A Clinico-Pathological Analysis of over 2500 Specimens and Biopsies. Journal of Urology, 74, 498-521. https://doi.org/10.1016/S0022-5347(17)67309-9

[13] Gopalan, A., Sharp, D.S., Fine, S.W., Tickoo, S.K., Herr, H.W., Reuter, V.E. and Olgac, S. (2009) Urachal Carcinoma. The American Journal of Surgical Pathology, 33, 659-668. https://doi.org/10.1097/PAS.0b013e31819aa4ae

[14] Dadhania, V., Czerniak, B. and Guo, C.C. (2015) Adenocarcinoma of the Urinary Bladder. American Journal of Clinical and Experimental Urology, 3, 51-63.

[15] Bates, A.W. and Baithun, S.I. (2000) Secondary Neoplasms of the Bladder Are Histological Mimics of Nontransitional Cell Primary Tumours: Clinicopathological and Histological Features of 282 Cases. Histopathology, 36, 32-40. https://doi.org/10.1046/j.1365-2559.2000.00797.x

[16] Wilson, T.G., Pritchett, T.R., Lieskovsky, G., Warner, N.E. and Skinner, D.G. (1991) Primary Adenocarcinoma of Bladder. Urology, 38, 223-226. https://doi.org/10.1016/S0090-4295(91)80349-C

[17] Zaghloul, M.S., Nouh, A., Nazmy, M., Ramzy, S., Zaghloul, A.S., Sedira, M.A. and Khalil, E. (2006) Long Term Results of Primary Adenocarcinoma of the Urinary Bladder: A Report on 192 Patients. Urologic Oncology, 24, 13-20. https://doi.org/10.1016/j.urolonc.2005.05.027

[18] Rogers, C.G., Palapattu, G.S., Shariat, S.F., Karakiewicz, P.I., Bastian, P.J., Lotan, Y., et al. (2006) Clinical Outcomes Following Radical Cystectomy for Primary Nontransitional Cell Carcinoma of the Bladder Compared to Transitional Cell Carcinoma of the Bladder. Journal of Urology, 175, 2048-2053. https://doi.org/10.1016/S0022-5347(06)00317-X

[19] Black, P.C., Brown, G.A. and Dinney, C.P. (2009) The Impact of Variant Histology on the Outcome of Bladder Cancer Treated with Curative Intent. Urologic Oncology, 27, 3-7. https://doi.org/10.1016/j.urolonc.2007.07.010

[20] Porten, S.P., Willis, D. and Kamat, A.M. (2014) Variant Histology, Role in Management and Prognosis of Nonmuscle Invasive Bladder Cancer. Current Opinion in Urology, 24, 517-523. https://doi.org/10.1097/MOU.0000000000000089

[21] Williams, C.R. and Chavda, K. (2015) En Bloc Robot-Assisted Laparoscopic Partial Cystectomy, Urachal Resection, and Pelvic Lymphadenectomy for Urachal Adenocarcinoma. Reviews in Urology, 17, 46-49.

[22] Makar, N. (1962) Some Observations on Pseudoglandular Proliferation in the Bilharzial Bladder. Acta Union Internationalis Contra Cancer, 18, 599-602.

[23] Fiter, L., Gimeno, F., Martin, L. and Gomez Tejeda, L. (1993) Signet-Ring Adenocarcinoma of the Bladder. Urology, 41, 30-33. https://doi.org/10.1016/0090-4295(93)90239-7

[24] Xu, W., Yu, B., Xu, T., Xu, Z., Cai, H. and Zou. Q. (2015) Chemotherapy for Primary Adenocarcinoma of the Urinary Bladder, Case Report. Advances in Pharmacoepidemiology \& Drug Safety, 4, 180.

[25] Logothetis, C.J., Samuels, M.L. and Ogden, S. (1985) Chemotherapy for Adenocar- 
cinomas of Bladder and Urachal Origin, 5-Fluorouracil, Doxorubicin, and Mitomycin-C. Urology, 26, 252-255. https://doi.org/10.1016/0090-4295(85)90121-9

[26] Siefker-Radtke, A.O., Gee, J., Shen, Y., Wen, S., Daliani, D., Millikan, R.E. and Pisters, L.L. (2003) Multimodality Management of Urachal Carcinoma: The M. D. Anderson Cancer Center Experience. Journal of Urology, 169, 1295-1298. https://doi.org/10.1097/01.ju.0000054646.49381.01

[27] Calabrò, F. and Sternberg, C.N. (2009) Neoadjuvant and Adjuvant Chemotherapy in Muscle-Invasive Bladder Cancer. European Urology, 55, 348-358. https://doi.org/10.1016/j.eururo.2008.10.016

[28] Clark, P.E. (2009) Neoadjuvant versus Adjuvant Chemotherapy for Muscleinvasive Bladder Cancer. Expert Review of Anticancer Therapy, 9, 821-830. https://doi.org/10.1586/era.09.36

[29] Dahm, P. and Gschwend, J.E. (2003) Malignant Non-Urothelial Neoplasms of the Urinary Bladder: A Review. European Urology, 44, 672-681. https://doi.org/10.1016/S0302-2838(03)00416-0

[30] Ghoneim, M.A., El-Mekresh, M.M., El-Baz, M.A., El-Attar, I.A. and Ashamallah, A. (1997) Radical Cystectomy for Carcinoma of the Bladder, Critical Evaluation of the Results in 1026 Cases. Journal of Urology, 158, 393-399. https://doi.org/10.1016/S0022-5347(01)64487-2

[31] Herr, H.W., Bochner, B.H., Sharp, P., Dalbagni, G. and Reuter, V.E. (2007) Urachal Carcinoma, Contemporary Surgical Outcomes. Journal of Urology, 178, 74-78. https://doi.org/10.1016/j.juro.2007.03.022

[32] John, N., Guido Sauter, E., Epstein, J.I. and Sesterhenn, I.A. (2004) Pathology and Genetics of Tumours of the Urinary System and Male Genital Organs. World Health Organization Classification of Tumours, International Agency for Research on Cancer (IARC), Lyon.

[33] Amin, M.B., Edge, S., Greene, F.L., Byrd, D.R., Brookland, R.K., Washington, M.K., et al. (2017) AJCC Cancer Staging Manual. 8th Edition, Springer International Publishing, Switzerland.

[34] Eisenhauera, E.A., Therasseb, P., Bogaertsc, J., Schwartzd, L.H., Sargente, D., Ford, R., et al. (2009) New Response Evaluation Criteria in Solid Tumours, Revised RECIST Guideline (Version 1.1). European Journal of Cancer, 45, 228-247. https://doi.org/10.1016/j.ejca.2008.10.026

[35] Korkes, K., Presti, M., Korkes, F. and Castro, M.G. (2009) Long-Term Follow-Up of Advanced Bladder Adenocarcinoma. Einstein, 7, 485-487.

[36] Johansson, S.L. and Cohen, S.M. (1997) Epidemiology and Etiology of Bladder Cancer. Seminars in Surgical Oncology, 13, 291-298.

https://doi.org/10.1002/(SICI)1098-2388(199709/10)13:5<291::AID-SSU2>3.0.CO;2 $\underline{-8}$

[37] El Bolkainy, M.N., Mokhtar, N.M., Ghoneim, M.A. and Hussein, M.H. (1981) The Impact of Schistosomiasis on the Pathology of Bladder Carcinoma. Cancer, 48, 2643-2648.

https://doi.org/10.1002/1097-0142(19811215)48:12<2643::AID-CNCR2820481216> 3.0.CO;2-C

[38] Zaghloul, M.S., Awwad, H.K., Omar, S., et al. (1992) Postoperative Radiotherapy of Carcinoma in Bilharzial Bladder. Improved Disease-Free Survival through Improving Local Control. International Journal of Radiation Oncology, Biology, Physics, 22, 511-517. https://doi.org/10.1016/0360-3016(92)90005-3 
[39] El-Bolkainy, M.N., Ghoneim, M.A. and Mansour, M.A. (1972) Carcinoma of the Bilharzial Bladder in Egypt, Clinical and Pathological Features. British Journal of Urology, 44, 561-570. https://doi.org/10.1111/j.1464-410X.1972.tb10123.x

[40] Mostofi, F.K. (1954) Potentialities of Bladder Epithelium. Journal of Urology, 71, 705-714. https://doi.org/10.1016/S0022-5347(17)67849-2

[41] El-Bolkainy, M.N. (1983) Schistosomiasis and Bladder Cancer. In: Cohen, S.M. and Bryan, C.T., Eds., The Pathology of Bladder Cancer, CRC Press, Boca Raton, Vol. 1, 57-89.

[42] Young, J.L., Percy, C.L., Asir, A., Berg, J.W., Gusano, M.M., Gloeckler, L.A., Horm, J.W., Lourie, W.I., Pollack, E.S. and Shambaugh, E.M. (1981) The SEER Program, Cancer Incidence and Mortality, 1973-77. Nati. Cancer Inst. Monogr., 57, 1-1082.

[43] Jacobo, E., Loening, S., Schmidt, J.D. and Culp, D.A. (1977) Primary Adenocarcinoma of the Bladder. Journal of Urology, 17, 54-56. https://doi.org/10.1016/S0022-5347(17)58335-4

[44] Bartsch, G.C., Kuefer, R., Gschwend, J.E., de Petriconi, R., Hautmann, R.E. and Volkmer, B.G. (2007) Hydronephrosis as a Prognostic Marker in Bladder Cancer in a Cystectomy-Only Series. European Urology, 51, 690-697.

https://doi.org/10.1016/j.eururo.2006.07.009

[45] Lin, H.Y., Wang, S.Z., Chen, J.X., Chen, L.W. and Xiao. J. (2011) The Prognostic Value of Hydronephrosis in Bladder Cancer Treated by Radical Cystectomy. Urologia, 78, 17-21. https://doi.org/10.5301/RU.2011.6444

[46] Dandekar, N.P., Dalal, A.V., Tongaonkar, H.B. and Kamat, M.R. (1997) Adenocarcinoma of Bladder. European Journal of Surgical Oncology, 23, 157-160. https://doi.org/10.1016/S0748-7983(97)80012-1

[47] Holmang, S., Borghede, G. and Johansson. S.L. (1997) Primary Signet-Ring Cell Carcinoma of the Bladder: A Report on 10 Cases. Scandinavian Journal of Urology and Nephrology, 31, 145-148. https://doi.org/10.3109/00365599709070320

[48] Yanagihara, Y., Tanji, N., Miura, N., Shirato, A., Nishimura, K., Fukumoto, T., Azuma, K., Miyauchi, Y., Kikugawa, T. and Yokoyama, M. (2013) Modified FOLFOX6 Chemotherapy in Patients with Metastatic Urachal Cancer. Chemotherapy, 59, 402-406. https://doi.org/10.1159/000362400

[49] Fan, F.S. and Yang, C.-F. (2018) Advanced Primary Nonurachal Adenocarcinoma of Urinary Bladder Responding to Modified FOLFOX6 and Capecitabine: A Case Report. Oxford Medical Case Reports, 8, 256-260. https://doi.org/10.1093/omcr/omy050 